\title{
Pentacene-Based Organic Thin Film Transistors for Ammonia Sensing
}

\author{
Hsiao-Wen Zan, Wu-Wei Tsai, Yen-ren Lo, Yu-Mei Wu, and Yuh-Shyong Yang
}

\begin{abstract}
Non-invasive ammonia sensors are attractive alternatives for the diagnoses of a variety of chronic diseases such as liver cirrhosis and renal failure. A low cost pentacene-based organic thin film transistor (OTFT) fabricated by a novel and simple process was demonstrated to be highly sensitive and specific for ammonia gas. Various measurement parameters that reflected OTFT device characteristics for ammonia detection were investigated. Significant variations of the turn-on current, intrinsic mobility, and threshold voltage $\left(V_{t h}\right)$ were observed while subthreshold swing $(S . S$.) was almost unchanged to the alteration of ammonia concentration. The OTFT device detected $0.5 \sim 5 \mathrm{ppm}$ concentration ammonia gas at room temperature, which is in the critical range that can distinguish between healthy person and paticents with liver cirrhosis and renal failure. The sensitivity of the device was further enhanced following a simple UV irradiation treatment to modify the functional groups on poly(methyl methacrylate) (PMMA) dielectric layer. Possible interference for ammonia detection such as humidity effect and selectivity among nitrogen, alcohol, carbon dioxide, acetone, methane and ammonia were also examined. We concluded that the proposed pentacene-based OTFT is a promising device for the future application in non-invasive medical diagnoses.
\end{abstract}

Index Terms-Ammonia sensor, non-invasive, OTFT, pentacene.

\section{INTRODUCTION}

A NALYZING the chemical composition of human breath helps people to examine their health conditions. It is a convenient method for a non-invasive diagnosis of disease and has been used for centuries [1]. More than 200 organic or inorganic gaseous molecules [2] were examined in human breath. These gaseous species were produced through normal physiological processes or pathological conditions such as gastric ulcer, liver disease, cancer, or renal failure. At present, few gas

Manuscript received August 31, 2010; revised January 22, 2011; accepted February 15, 2011. Date of publication March 03, 2011; date of current version February 01, 2012. This work was supported in part by the National Science Council (NSC97-2221-E-007-018-MY3, 97-2627-B-009-009 and 99-2627-B009-004), and in part by the National Nano Device Laboratories (P96-1A-021). The associate editor coordinating the review of this manuscript and approving it for publication was Prof. Aime Lay-Ekuakille.

H.-W. Zan, W.-W. Tsai, and Y.-M. Wu are with Department of Photonics and Institute of Electro-Optical Engineering and Department of Photonics and Display Institute, National Chiao Tung University, Hsinchu 300, Taiwan (e-mail: hsiaowen@ mail.nctu.edu.tw; wuweitsai@gmail.com; cetacea-mui@ hotmail.com).

Y.-R. Lo and Y.-S. Yang are with the Department of Biological Science and Technology, National Chiao Tung University, Hsinchu 300, Taiwan (e-mail: itismevicugna@hotmail.com; ysyang@faculty.nctu.edu.tw).

Color versions of one or more of the figures in this paper are available online at http://ieeexplore.iee.org.

Digital Object Identifier 10.1109/JSEN.2011.2121901 sensors are specific to only a single gas. However, high specificity of gas sensing can still be achieved by using an array of gas sensing devices that monitor a different spectrum of gaseous species. Table I shows some unusual breath odors mainly caused by the increase of specific gaseous molecules that are associated with important diseases [3]-[7]. A gas sensor that can monitor the unusual concentrations of these specific molecules in human breath would be very useful for the development of non-invasive diagnosis of these diseases.

Ammonia is an important indicator for uremia and chronic liver disease (Table I). Breath ammonia level is significantly higher in cirrhotic patients $(0.745 \mathrm{ppm})$ than that in normal person $(0.278 \mathrm{ppm})$ [8]. Patients who have renal failure even exhale $4.8 \mathrm{ppm}$ ammonia in their breath [5]. It is suggested that suitable ammonia sensors would be important. Current ammonia sensors such as polyaniline sensors, metal oxide sensors, catalytic sensors, and optical analyzers suffer from disadvantages such as high operation temperature, low sensitivity and high cost [9], [10]. Adequate ammonia sensor for biomedical application should be able to detect ammonia from around 0.5 to $5 \mathrm{ppm}$ at room temperature as described above for cirrhotic [8] and renal failure patients [5]. Other diseases that are diagnosed with human breath included $H$. pylori infection [6] with C13 labeled carbon dioxide $\left({ }^{13} \mathrm{CO}_{2}\right)$ and diabetes using gas-sensitive LAPS (Light Addressable Potentiometric Sensors) [11]. However, both cases are limited in hospitals or medical centers for the readout of the sensing signals required expensive instruments. Facing with the ageing society and urgent need for personalized medicine, it is essential to develop cheap and portable gas sensors for patients and to trace their conditions at home.

Organic thin-film transistors (OTFTs) were reported to be a non-invasive, inexpensive, portable and disposable diagnostic device because of its low cost fabrication process and high sensitivity to gas molecules [12]. The molecular active channel in OTFTs enables the devices to exhibit rapid response in both gaseous [13] and aqueous [14] sensing environment. It is proposed that the gas molecules penetrate organic active layer through grain boundaries and diffuse into channel region to react with carriers [15], [16]. As a result, the sensitivity is high and is strongly dependent on the morphology and the grain boundary density of the organic film [17]. OTFTs provide multiple sensing parameters such as field-effect mobility, turn-on conductivity, turn-off conductivity, threshold voltage $\left(V_{\text {th }}\right)$ and subthreshold swing (S.S.) [18]. Also, OTFTs with different active layer materials can form an OTFT array to provide sensing selectivity through a sensing map [12]. Some approaches have been demonstrated to increase the sensitivity and selectivity of OTFT gas sensor by changing the functional 
TABLE I

DisEASES ASSOCIATED WITH UNUSUAL BREATH OdORS

\begin{tabular}{|c|c|c|}
\hline $\begin{array}{c}\text { Breath component as a } \\
\text { disease marker }\end{array}$ & Diseases & References \\
\hline Acetone & Diabetes; lung cancer & {$[3],[4]$} \\
\hline Ammonia & $\begin{array}{c}\text { Uremia; Liver Cirrhosis } \\
\text { Renal failure }\end{array}$ & {$[5],[7]$} \\
\hline Butyric acid & Liver Cirrhosis & {$[7]$} \\
\hline Ethanethiol & Liver Cirrhosis & {$[7]$} \\
\hline Hydrogen sulfide & Periodontal disease & {$[7]$} \\
\hline Carbon dioxide & H. Pylori infection & {$[6]$} \\
\hline
\end{tabular}

TABLE II

COMPARISONS OF AMMONIA SENSORS IN THIS WORK AND IN OTHER REPORTS

\begin{tabular}{|c|c|c|c|c|}
\hline Principle & $\begin{array}{c}\text { Low } \\
\text { detection } \\
\text { limit }\end{array}$ & Response time & $\begin{array}{l}\text { Temperature } \\
\text { range }\end{array}$ & Remarks [reference] \\
\hline Optical gas sensors/Nessler & $90 \mathrm{ppb}$ & $\sim 1 \mathrm{~min}$ & $37^{\circ} \mathrm{C}$ & To detect in water[22] \\
\hline Optical gas sensors/ Coulorometric & $1 \mathrm{ppt}$ & $\sim 5 \mathrm{~min}$ & $\mathrm{~N} / \mathrm{A}$ & Expensive setup[23] \\
\hline $\begin{array}{l}\text { Optical gas sensors/ } \\
\text { Porous Silicon }\end{array}$ & $180 \mathrm{ppb}$ & $\mathrm{N} / \mathrm{A}$ & Room temperature & Expensive setup[24] \\
\hline Catalytic metal/Palladium & $1 \mathrm{ppm}$ & $\sim 1$ min & Up to $600^{\circ} \mathrm{C}$ & Low selectivity[25] \\
\hline Catalytic metaV/Polyaniline & $1 \mathrm{ppm}$ & $\sim 3 \mathrm{~min}$ & Up to $150^{\circ} \mathrm{C}$ & $\begin{array}{l}\text { Irreversible reactions } \\
{[26,27]}\end{array}$ \\
\hline OTFT/P3HT & $10 \mathrm{ppm}$ & $2 \sim 3 \min$ & Room temperature & Low sensitivity[28] \\
\hline $\begin{array}{l}\text { Our proposed/pentacene-based } \\
\text { OTFT }\end{array}$ & $0.5 \mathrm{ppm}$ & $\sim 500 \mathrm{sec}$ & Room temperature & $\begin{array}{l}\text { Low cost and high } \\
\text { sensitivity }\end{array}$ \\
\hline
\end{tabular}

group, the grain structure of organic layer [19], or varying the device dimension [20]. The OTFT response to ammonia gas [21] has been briefly mentioned. However, the potential and its feasibility for using OTFTs as non-invasive diagnostic sensor deserve further investigation. In this report, for the first time, we fabricated OTFTs that were suitable as an ammonia sensor for monitoring cirrhotic and renal failure patients. Table II compares the ammonia sensors of this work and of others reports [22]-[28]. Up to date, only optical sensing systems, which require expensive setup, exhibit a sensitivity in parts-per-billion (ppb) or even parts-per-trillion (ppt) regime. Electrical ammonia sensors, like poly(3-hexylthiophene)(P3HT)-based OTFT, exhibit sensitivity higher than $10 \mathrm{ppm}$. Electrical sensors based on catalytic metals exhibit sensitivity as $1 \mathrm{ppm}$, however, the sensors have to be operated at a temperature higher than $150^{\circ} \mathrm{C}$. Our proposed pentacene-based OTFT, particularly the UV-treated pentacene-based OTFT, exhibits sensitivity as 0.5 $\mathrm{ppm}$ at room temperature.

\section{EXPERIMENT}

\section{A. Preparation of OTFTs}

OTFTs were fabricated on silicon substrates as shown in Fig. 1(a)-(d). A highly doped p-type silicon wafer with 100-nm-thick $\mathrm{SiO}_{2}$ was used as the gate electrode and gate insulator, respectively. PMMA [poly(methyl methacrylate)] obtained from MicroChem Corp (with a molecular weight of 95000) was used as the buffer layer to modify $\mathrm{SiO}_{2}$ dielectric surface. PMMA dissolved in anisole at $10 \mathrm{wt} \%$ was spun onto $\mathrm{SiO}_{2}$ dielectric surface at a speed of approximately $8000 \mathrm{rpm}$ for 40 seconds. Then, the $\mathrm{SiO}_{2}$ with PMMA was transferred to a hot-plate and annealed at $90^{\circ} \mathrm{C}$ for 30 minutes. The thickness of PMMA was estimated by capacitance measurement Agilent $\mathrm{HP} 4284$. The capacity of PMMA/ $\mathrm{SiO}_{2}$ dielectric was about 23-24 nF/ $\mathrm{cm}^{2}$. Part of the $\mathrm{SiO}_{2}$ with PMMA was exposed to UV-light (175-285 nm and irradiation intensity of 0.043 $\mathrm{mW} / \mathrm{cm}^{2}$ for 60 seconds; Jelight Company, GLS-144 UV 
(a)

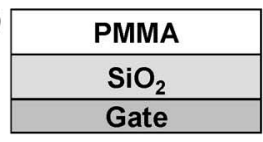

(b)

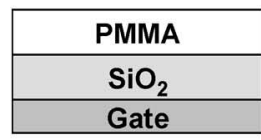

(c)

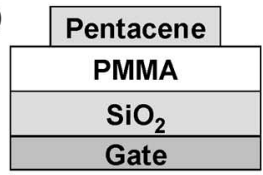

(d)

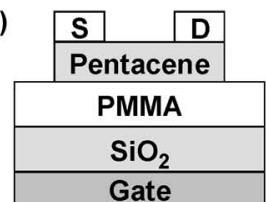

(e)

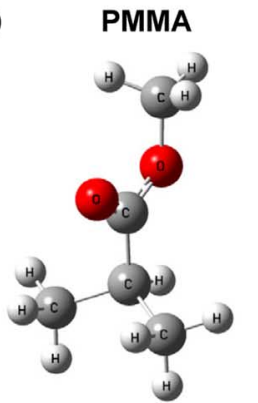

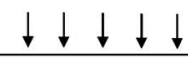

UV-treated PMMA $\mathrm{SiO}_{2}$ Gate

Pentacene UV-treated PMMA $\mathrm{SiO}_{2}$ Gate

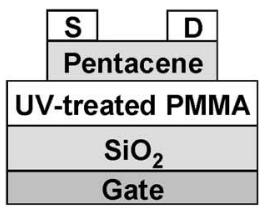

UV-treated PMMA

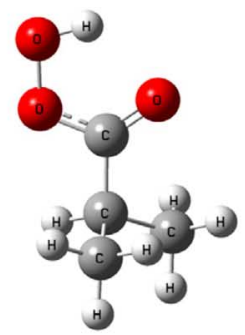

Fig. 1. Fabrication and structure of pentacene-based OTFT. (a) A highly doped p-type silicon wafer with 100 -nm-thick $\mathrm{SiO}_{2}$ was used as the gate electrode and gate insulator, respectively. (b) Part (right) of the $\mathrm{SiO}_{2}$ with PMMA was exposed to UV-light (Jelight Company, GLS-144 UV Lamp). (c) Both UV-treated PMMA (right) and untreated (left) PMMA dielectrics were transferred into a vacuum chamber for the deposition of 100-nm-thick pentacene film. The pentacene (99.9\% pure) was evaporated through a shadow mask to form the active layer. (d) After the formation of a 100-nm-thick pentacene, 100-nm-thick gold was deposited through the shadow mask to form source/drain contacts. (e) The functional end group of standard and UV-treated PMMA molecules.

Lamp). It is proposed that UV light treatment modified the PMMA functional end-groups from - $\mathrm{COOCH} 3$ to $-\mathrm{COOOH}$ (Fig. 1(e)) [29], [30]. After UV treatment, the dipole moment of PMMA end-groups is increased [30].

Both UV-treated PMMA and untreated (standard, STD) PMMA dielectrics were transferred into a vacuum chamber for the deposition of 100-nm-thick pentacene film. The pentacene (99.9\% pure, obtained from Aldrich without purification) was evaporated through a shadow mask to form the active layer. The deposition rate was set at $0.5 \AA / \mathrm{s}$ with the substrate temperature as room temperature and the pressure at around $1 \times 10^{-6}$ Torr. After the formation of a 100 -nm-thick pentacene, 100-nm-thick gold was deposited through the shadow mask to form source/drain contacts. Schematic cross-sectional view of STD- and UV-Treated OTFTs (UV-OTFTs) are shown in Fig. 1(d). Fig. 2(a) also shows the flowchart for the fabrication processes.

\section{B. Electric Characterization of OTFTs}

Electric properties of OTFT devices were measured by using a semiconductor analyzer (Keithley 4200-SCS) in a sealed chamber. The inside total chamber-volume was $42 \mathrm{~L}$. The sealed chamber with measurement system is shown in Fig. 3. To begin a measurement, the chamber was initially vacuumed to be less than 1 torr and then purged with high-purity (99.99\%) nitrogen $\left(\mathrm{N}_{2}\right)$ to $1 \mathrm{~atm}$. Then, $\mathrm{NH}_{3}$ gas (Airproduct, $>99 \%$ ) was injected through a mass flow controller (Brooks, MFC $5850 \mathrm{E}, \mathrm{USA}$ ) into the chamber during a controlled time period. The concentration of $\mathrm{NH}_{3}$ gas in the chamber was calculated according to the chamber dimension as ppm unit $(\mathrm{mg} / \mathrm{L})$.

\section{Moisture Control}

The relative humidity $(\mathrm{RH})$ in the chamber was controlled by firstly introducing water vapor to increase $\mathrm{RH}$ then pumping down to keep the $\mathrm{RH}$ at $50 \%$. The $\mathrm{RH}$ was measured by the humidity gauge (WISEWIND, 5334, China) inside the sealed chamber. The flowchart for measurement characterization is shown in Fig. 2(b).

\section{RESUlTS AND DISCUSSION}

\section{A. Initial Characteristics of STD and UV-OTFTs}

The transfer characteristics of STD- and UV-OTFTs are compared in Fig. 4. Though the current-voltage model of OTFTs is still not well-developed, formula borrowed from MOSFET are widely used to analyze OTFT characteristics from macroscopic point of views [31]-[33]. In linear region, drain current $\left(I_{\mathrm{d}}\right)$ can be expressed as (1) [18], [31]

$$
I_{d}=\frac{W \mu}{L} C_{i}\left[\left(V_{g}-V_{t h}\right) \cdot V_{d}-\frac{1}{2} V_{d}^{2}\right]
$$

where $C_{\mathrm{i}}$ is capacitance per unit area of the insulating layer, $\mu$ is the field-effect mobility, and $V_{\text {th }}$ is the threshold voltage. When $V_{\mathrm{d}} \ll\left(V_{\mathrm{g}}-V_{\mathrm{th}}\right)$, (1) can be simplified as (2) and $V_{\mathrm{th}}$ can be extracted by fitting (2) with the linear region drain current

$$
I_{d}=\frac{W \mu}{L} C_{i}\left(V_{g}-V_{t h}\right) \cdot V_{d}
$$

Then, linear-region transconductance can be defined as

$$
g_{m}=\left[\frac{\partial I_{d}}{\partial V_{g}}\right]_{V_{d}=\text { constant }}=\frac{W \mu}{L} C_{i} \cdot V_{d}
$$

Field-effect mobility is then extracted from the transconductance maximum by using (4) [18]

$$
\mu=\frac{g_{m}}{\left(\frac{W}{L} C_{i} V_{d}\right)}
$$

Typical parameters extracted from transfer characteristics are listed in inset of Fig. 4. $V_{\text {th }}$ of UV-OTFTs was more positive 

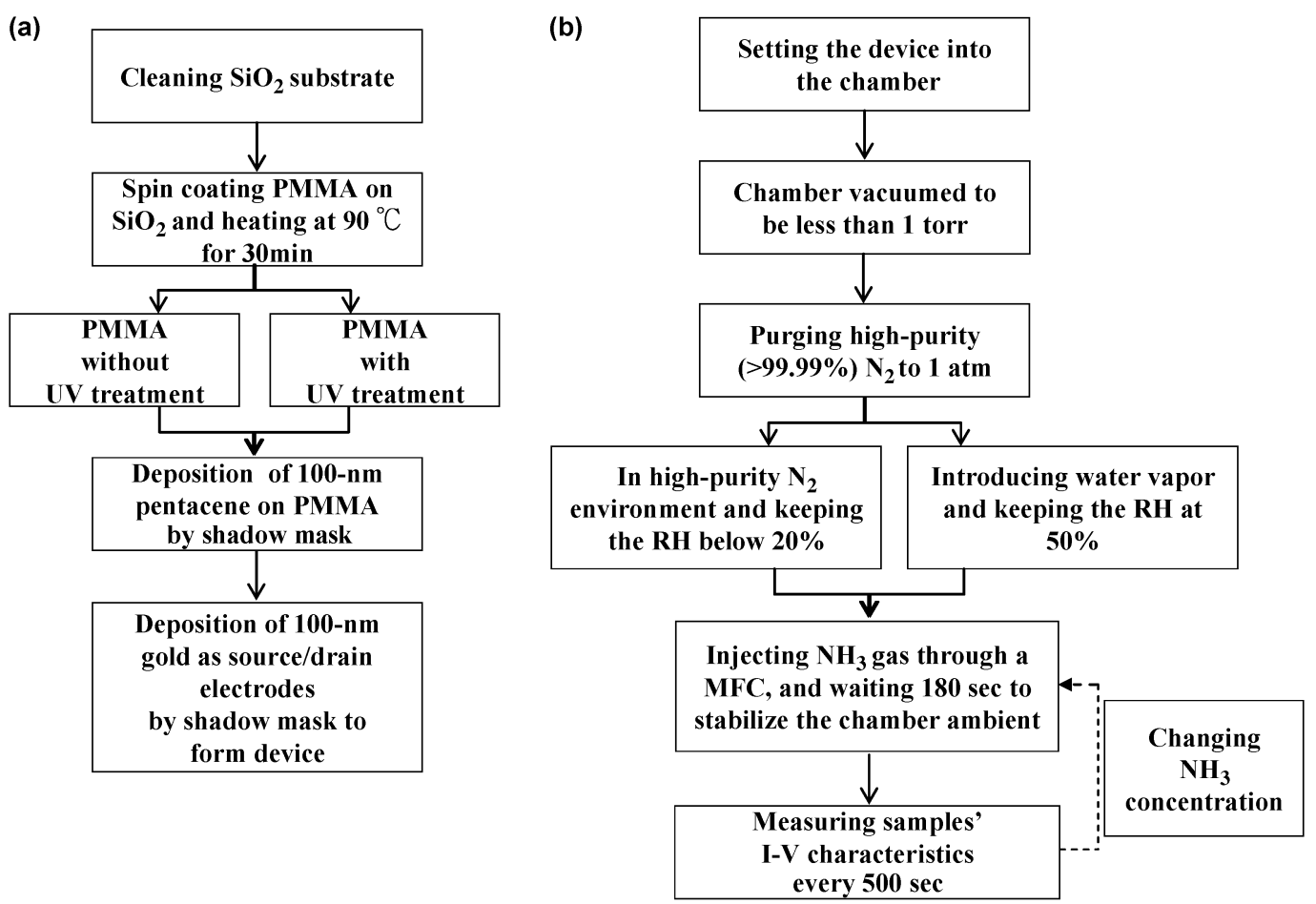

Fig. 2. (a) Flowchart for the fabrication processes and (b) for the measurement characterization.

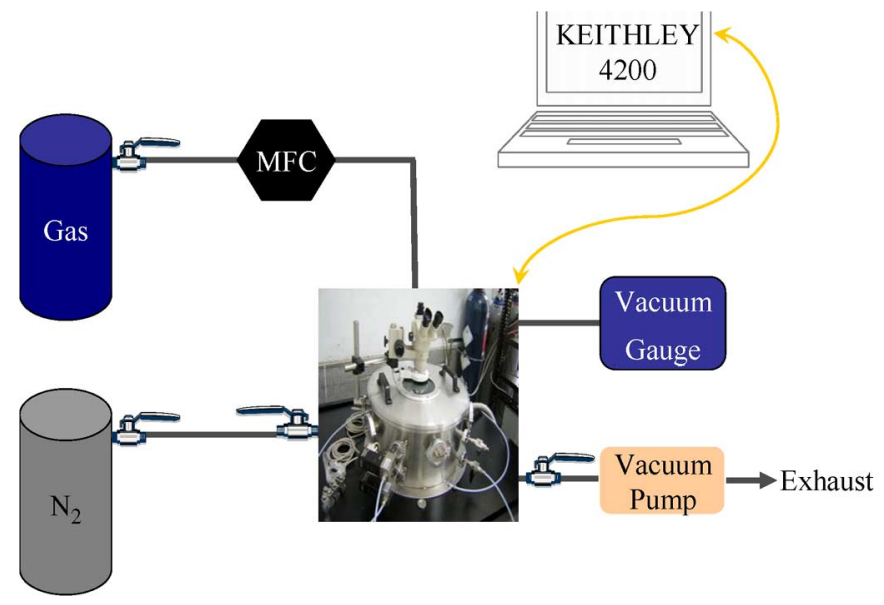

Fig. 3. Gas sensing system. The chamber holder is surrounded by the 5 probing-stages and the injected gas is controlled by mass flow controller (MFC). Before starting the gas sensing experiment, we used the mechanical pump to pump down the chamber at a pressure less than 1 torr. Then we used the high-purity nitrogen gas (99.99\%) to purge the chamber to a pressure about $1 \mathrm{~atm}$ and kept the chamber in the low moisture and oxygen. Then, we introduced ammonia gas through the mass flow controller.

than that of STD-OTFTs. A plausible explanation is that the UV-treated PMMA produces excess negatively charges that enhanced the accumulation of holes. Accordingly, S.S. was enlarged and the $V_{\text {th }}$ was positively shifted. These results are consistent with previously reported data [30], [34], [35]. Both standard and UV-treated devices exhibited a mobility around 0.3 $\mathrm{cm}^{2} / \mathrm{Vs}$ (inset table of Fig. 4), indicating that the pentacene film structure and the carrier transport were not significantly affected following UV treatment. The mobility of pentacene-

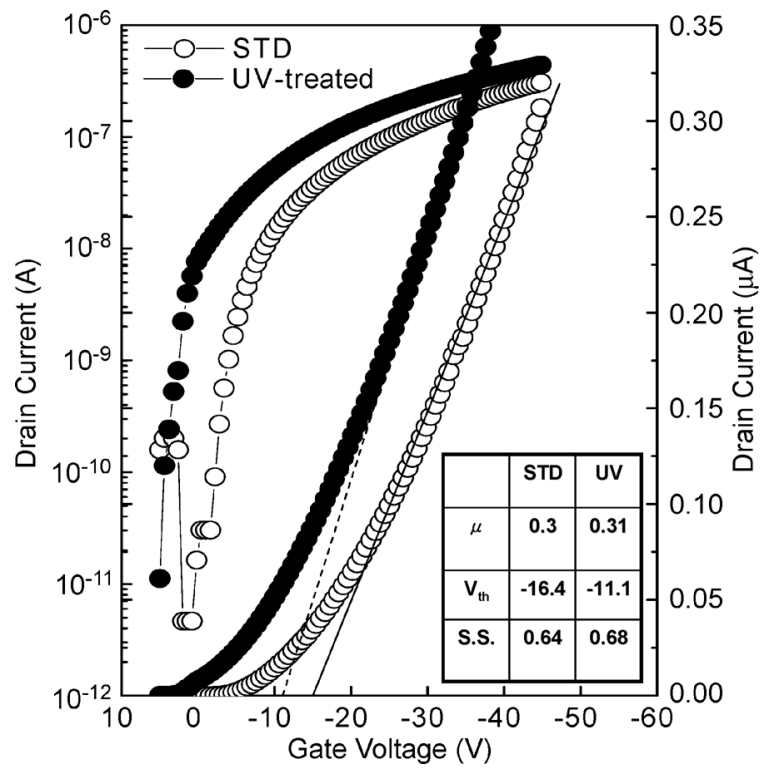

Fig. 4. Transfer characteristics of STD and UV-OTFTs. Drain current $\left(I_{\mathrm{d}}\right)$ was measured at a constant drain voltage $\left(V_{\mathrm{d}}=-3 \mathrm{~V}\right)$ while gate bias $\left(V_{\mathrm{g}}\right)$ sweeps from 5 to -45 volts. Field effect mobility and threshold voltage were calculated in the linear regime $\left(V_{\mathrm{d}}=-3 \mathrm{~V}\right)$ defined by standard metal-oxide-semiconductor FET model. S.S was also extracted from the transfer characteristics. The extracted typical electrical parameters [mobility $\left(\mu\left(\mathrm{cm}^{2} / \mathrm{Vs}\right)\right)$, threshold voltage $\left(V_{\text {th }}(\mathrm{V})\right)$, subthreshold swing $(S . S .(\mathrm{mV} /$ decade $\left.))\right]$ are summarized in the inset table.

based OTFT is much smaller than that of single crystal silicon MOSFET and leads to a small output current. To deliver a reasonable current with an obvious sensing response, one can increase the device channel width to channel length ratio (W/L) to enlarge the output current as well as the sensing response. 


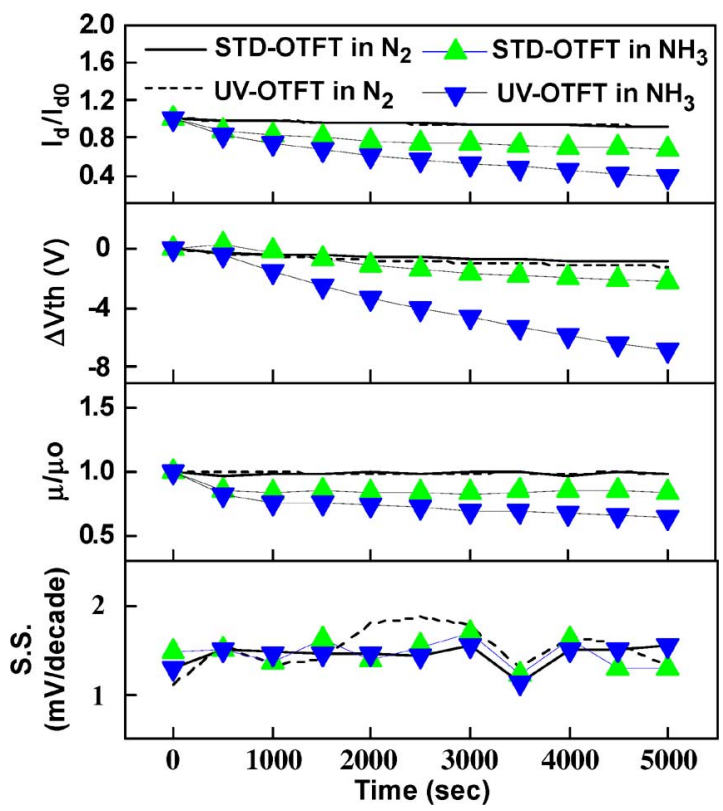

Fig. 5. Ammonia sensing responses of OTFTs with multi-parameters. The multi-parameters, $I_{d} / I_{d 0}, \Delta V_{\mathrm{th}}, \mu / \mu_{0}$, and $S . S$. of STD- and UV-OTFTs are plotted as a function of time in $\mathrm{NH}_{3}(1 \mathrm{ppm})$ and $\mathrm{N}_{2}$ ambiences. Each data point was extracted from the transfer characteristics measured at $V_{\mathrm{g}}=-40 \mathrm{~V}$, $V_{\mathrm{d}}=-3 \mathrm{~V}$ with the interval of 500 secs within a total sensing time of 5000 secs.

A signal read-out circuit can also be designed to amplifier the sensing signal.

\section{B. Ammonia Sensing Response of STD- and UV-OTFTs}

The responses of STD- and UV-OTFTs to nitrogen ambient and to 1-ppm ammonia gas in nitrogen ambient as a function of sensing time were determined, respectively. Four typical parameters (turn-on current, intrinsic mobility, $V_{t h}$, and $S . S$.) were extracted and plotted as a function of sensing time as shown in Fig. 5. Both STD- and UV-OTFTs keep almost unchanged characteristics in nitrogen ambient. In the presence of 1-ppm ammonia gas, significant variations of the turn-on current, intrinsic mobility, and $V_{t h}$ were observed while S.S. was almost unchanged. The turn-on current variation $\left(I_{\mathrm{d}} / I_{\mathrm{d} 0}\right)$, according to (2), is affected by both threshold voltage shift $\left(\Delta V_{\mathrm{th}}\right)$ and mobility variation $\left(\mu / \mu_{0}\right)$, where $I_{\mathrm{d} 0}$ and $\mu_{0}$ are the initial drain current and the initial field-effect mobility. S.S. is usually referred to the interface trap density [29] and constant S.S. indicated that 1-ppm $\mathrm{NH}_{3}$ did not drastically change the interface trap density of the device. When ammonia molecules diffuse into the active layer through grain boundaries, the decrease in mobility may result from the increase of scattering centers or the increase of energy barrier for charge transport [16]. It was reported that the polar molecules disturb charge transport in organic materials by increasing the amount of energetic disorder through charge-dipole interactions [36]. After exposing to 1-ppm ammonia gas for $1000 \mathrm{sec}$, mobility of STD-OTFT and UV-OTFT degraded about $20 \%$ and $30 \%$, respectively. This result implied that the increased dipole moment on PMMA surface following UV treatment may facilitate the absorption of ammonia molecules.
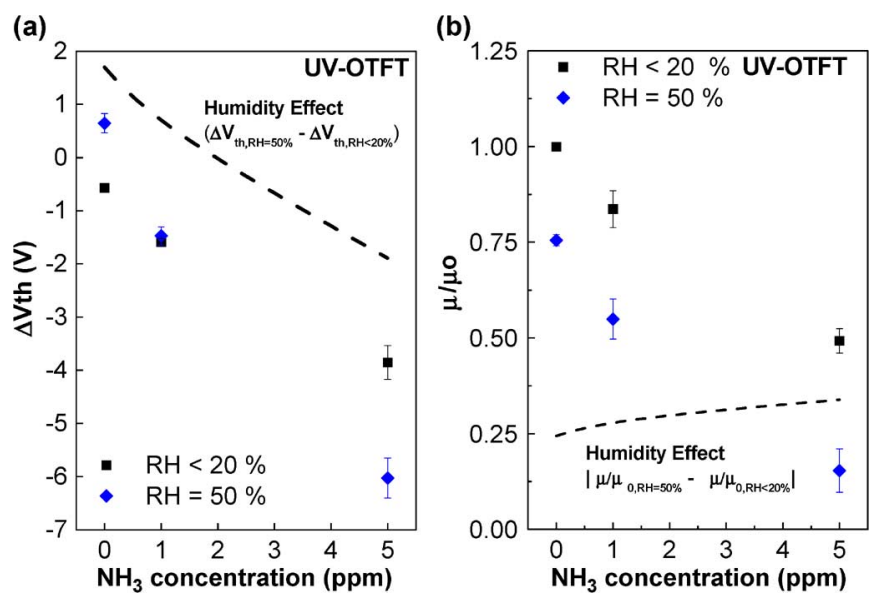

Fig. 6. Humidity effect on ammonia sensing using UV-OTFTs. (a) $\Delta V_{\text {th }}$ and (b) $\mu / \mu_{0}$ of UV-OTFTs were determined when devices were exposed to different concentrations of $\mathrm{NH}_{3}$ gas with $\mathrm{RH}$ less than $20 \%$ or equal to $50 \%$. Humidity effect (shown as dashed lines) is defined as $\Delta V_{\mathrm{th}, \mathrm{RH}=50 \%}-\Delta V_{\mathrm{th}, \mathrm{RH}<20 \%}$ and $\mu / \mu_{0, \mathrm{RH}=50 \%}-\mu / \mu_{0, \mathrm{RH}<20 \%}$ for $\Delta V_{\text {th }}$ and $\mu / \mu_{0}$, respectively. The sensing time was fixed at $2000 \mathrm{sec}$. RH of the sealed sensing chamber was controlled at $\mathrm{RH}<20 \%$ and $\mathrm{RH}=50 \%$, respectively. Each data was the average of three independent measurements by using three samples with identical initial characteristics.

The influence of UV treatment on OTFT sensing behavior was much more pronounced when the $\Delta V_{\text {th }}$ was observed (Fig. 5). $\Delta V_{\text {th }}$ is usually referred to charge trapping in insulator or defect state creation in pentacene film [37], [38]. It has been observed that OTFTs exhibit significant $\Delta V_{\text {th }}$ due to defect state creation when OTFTs are continuously biased (bias-stress effect) [39], exposed to light [40], or exposed to moisture [41]. It is unlikely that this pronounced $\Delta V_{\text {th }}$ was due to the bias-stress effect in our experiment because both STD- and UV-OTFTs keep almost unchanged characteristics in nitrogen ambient. It is proposed that the ammonia molecules within pentacene/PMMA interface may interact with pentacene molecules to form positively-charged defect state and cause variation of $\Delta V_{\mathrm{th}}, I_{\mathrm{d}} / I_{\mathrm{d} 0}$, and $\mu / \mu_{0}$. After UV treatment, PMMA surface becomes more hydrophilic and may attract more ammonia.

\section{Humidity Effect on Ammonia Sensing}

Ammonia $\left(\mathrm{NH}_{3}\right)$ may become positively-charged ammonium ion $\left(\mathrm{NH}_{4}^{+}\right)$in the presence of water and may significantly affect the electric properties of the OTFTs. We thus investigated the effect of relative humidity ( $\mathrm{RH})$ on the ammonia sensing. In the nitrogen ambient and in the absence of ammonia, reversed RH effects (or water molecule doping effects) were observed for $\Delta V_{\text {th }}$ (Fig. 6(b)) and $\mu / \mu_{0}$ (Fig. 6(b)). This observation can be explained as following. The highly polar water molecules can induce more mobile charges in the organic semiconductor layer. Therefore, a large positive gate bias was needed to compensate for the holes in the channel and to turn off the device [41]. Scattering effect or the field screening effect may be the plausible mechanism to describe interactions between polar water molecules and carriers and can explain why carrier mobility was decreased at higher RH. The humidity effect $\left(\Delta V_{\mathrm{th}, \mathrm{RH}=50 \%}-\Delta V_{\mathrm{th}, \mathrm{RH}<20 \%}\right)$ on $\Delta V_{\mathrm{th}}$ is show as the dashed line in Fig. 6(a). Interestingly, increasing RH (from $20 \%$ to $50 \%$ ) and higher ammonia concentration 

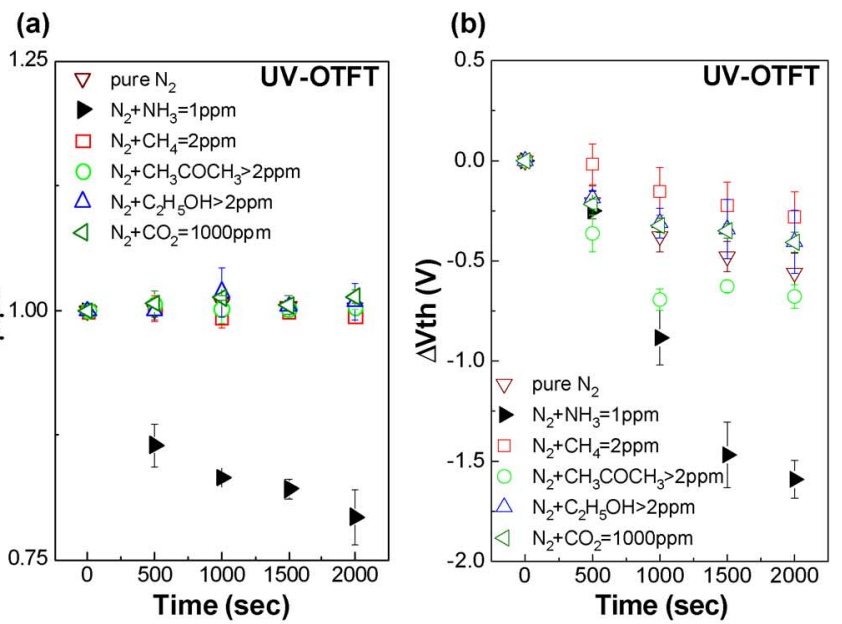

(c)
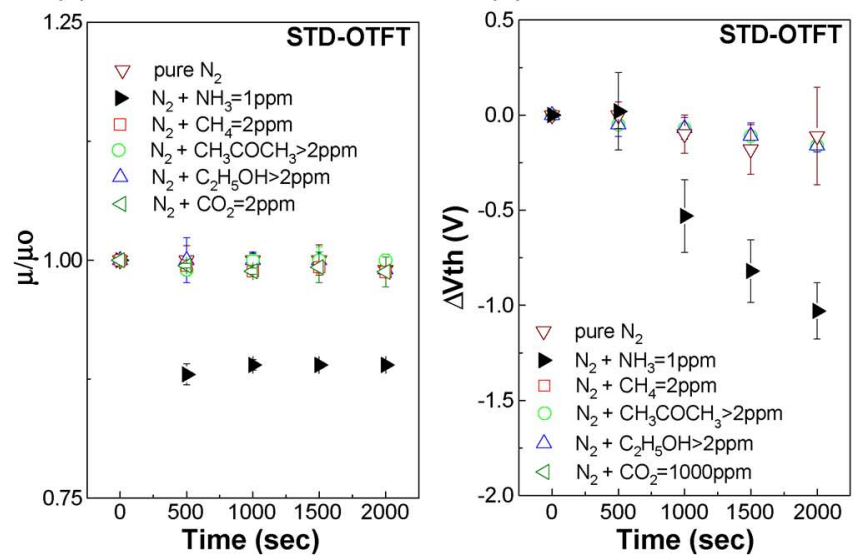

Fig. 7. Target dependent OTFT responses. (a) and (b) Variations of $\mu / \mu_{0}$ and $\Delta V_{\mathrm{th}}$ as a function of sensing time for UV-OTFTs. (c) and (d) Variations of $\mu / \mu_{0}$ and $\Delta V_{\text {th }}$ as a function of sensing time for STD-OTFTs. Each data was the average of three independent measurements.

made $\Delta V_{\text {th }}$ more negative. This observation was consistent with the formation of ammonium ions $\left(\mathrm{NH}_{4}^{+}\right)$in the presence of water molecules, which suppressed the water doping effect. However, $\mu / \mu_{0}$ of OTFT was not sensitive to the variation of $\mathrm{RH}\left(\mu / \mu_{0, \mathrm{RH}=50 \%}-\mu / \mu_{0, \mathrm{RH}<20 \%}\right.$, dashed line in Fig. 6(b)), probably because charged and polar molecule gave relatively similar effect on $\mu / \mu_{0}$. In this report, constant RH was maintained to avoid its interference on ammonia sensing. More detailed effects of RH on ammonia sensing are now under investigation.

\section{The Specificity of OTFTs}

The specificity of OTFT as gas sensor was further investigated with some gases that may exist in human breath or may interfere with ammonia sensing. The effects of carbon dioxide $\left(\mathrm{CO}_{2}\right)$, alcohol $\left(\mathrm{C}_{2} \mathrm{H}_{5} \mathrm{OH}\right)$, methane $\left(\mathrm{CH}_{4}\right)$, and acetone $\left(\mathrm{CH}_{3} \mathrm{COCH}_{3}\right)$ on $\mu / \mu_{0}$ and $\Delta V_{\text {th }}$ of UV- and STD-OTFTs were shown in Fig. 7(a), (b) and Fig. 7(c), (d), respectively. These gases were relatively inert to OTFTs and only ammonia caused significant variation in both $\mu / \mu_{0}$ and $\Delta V_{\mathrm{th}}$.

(a)

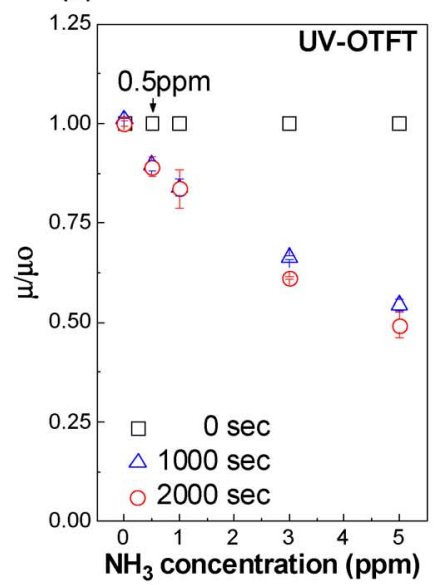

(c)

(b)

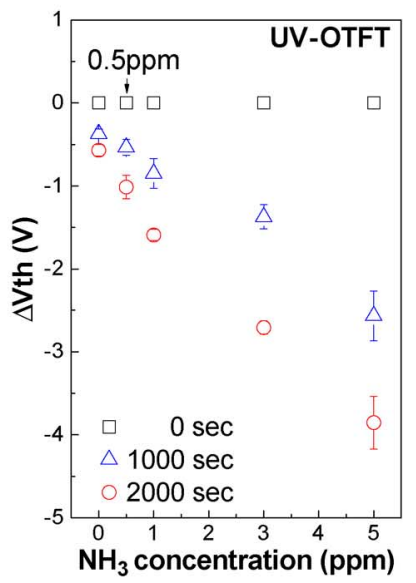

(d)

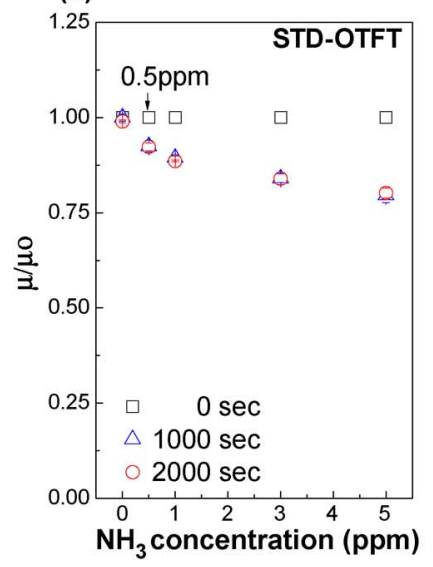

Fig. 8. Concentration dependent ammonia sensing response. (a) and (b) Variations of $\mu / \mu_{0}$ and $\Delta V_{\text {th }}$ as a function of ammonia concentration for UV-OTFTs. (c) and (d) Variations of $\mu / \mu_{0}$ and $\Delta V_{\text {th }}$ as a function of ammonia concentration for STD-OTFTs. Each data was the average of three independent measurements.

\section{E. Ammonia Concentration Dependent OTFT Sensing Response}

Both $\mu / \mu_{0}$ and $\Delta V_{\text {th }}$ of OTFTs were dependent on ammonia concentration as shown in Fig. 8. The variation of $\mu / \mu_{0}$ and $\Delta V_{\text {th }}$ to ammonia concentration indicated that the difference of less than $0.5 \mathrm{ppm}$ ammonia can be clearly distinguished by OTFTs. As a non-invasive diagnostic sensor for cirrhotic patients, it is necessary to monitor ammonia concentration at $0.5 \mathrm{ppm}$ or lower so that the breath samples between healthy person (breath ammonia level: $0.278 \mathrm{ppm}$ ) and a patient (breath ammonia level: $0.745 \mathrm{ppm}$ ) [8] can be distinguished. For the patients with renal failure, the target ammonia levels are approximately $1 \mathrm{ppm}$ (relieve) to $5 \mathrm{ppm}$ (dangerous) [7]. Our data shown in Fig. 8 demonstrated that both UV-OTFTs (Fig. 8(a), (b)) and STD-OTFTs (Fig. 8(c), (d)) can reach this requirement for ammonia sensitivity.

\section{CONCLUSION}

In conclusion, a pentacene-based OTFT was shown to be sensitive for ammonia sensing from 0.5 to $5 \mathrm{ppm}$, a critical range for the diagnosis of patients with chronic liver diseases and renal failure. This demonstrated that OTFT devices, which can be 
fabricated by simple and cheap process and exhibited channel length and width as large as several hundreds of microns, were useful as non-invasive biomedical sensors. The advantages of OTFT devices are on the contrary to inorganic MOSFET devices that require high fabrication cost and complicated fabrication process and the dimension of the devices has to be scaled down to the range of nanometers to increase the gas sensing sensitivity. The sensitivity and selectivity of OTFTs as gas sensor can be further improved by the modification of the PMMA dielectric layer and selecting suitable measuring parameters. Due to the simple fabrication processes of the devices, OTFTs are promising to be developed to a portable and disposable gas sensor.

\section{REFERENCES}

[1] R. B. Northrop, "Noninvasive instrumentation and measurement in medical diagnosis," in The Biomedical Engineering, 1st ed. Boca Raton, FL: CRC Press, 2002.

[2] J. P. Conkle, B. J. Camp, and B. E. Welch, "Trace composition of human respiratory gas," Arch. Enuviron. Health, vol. 30, pp. 290-295, 1975.

[3] S. E. Ebeler, A. J. Clifford, and T. Shibamo, "Quantitative analysis by gas chromatography of volatile carbonyl compounds in expired air from mice and human," J. Chromatogr. B., vol. 702, p. 211, 1997.

[4] C. Grote and J. Pawliszyn, "Solid-phase microextraction for the analysis of human breath," Anal. Chem., vol. 69, p. 587, 1997.

[5] S. Davies, P. Spanel, and D. Smith, "Quantitative analysis of ammonia on the breath of patients in end-stage renal failure," Kidney Int., vol. 52, p. 223, 1997.

[6] D. W. Chey and C. Y. Wong et al., "American College of Gastroenterology guideline on the management of helicobacter pylori infection," Amer. J. Gastroenterol., vol. 102, pp. 1808-1825, 2007.

[7] A. Manolis, "The diagnostic potential of breath analysis," Clin. Chem., vol. 29, p. 5, 1983.

[8] C. Shimamoto, I. Hirata, and K. Katsu, "Breath and blood ammonia in liver cirrhosis," Hepato-Gastroenterol., vol. 47, p. 443, 2000.

[9] G. K. Prasad, T. P. Radhakrishnan, D. Sravan Kumar, and M. G. Hanashyam Krishna, "Ammonia sensing characteristics of thin film based on polyelectrolyte templated polyaniline," Sens. Actuators B., vol. 28, pp. 626-631, 2005, 106.

[10] B. Timmer, W. Olthuis, and A. V. D. Berg, "Ammonia sensors and their applications-A review," Sens. Actuators B., vol. 107, pp. 666-677, 2005.

[11] Q. Zhang, P. Wang, J. Li, and X. Gao, "Diagnosis of diabetes by image detection of breath using gas-sensitive laps," Biosens. Bioelectron., vol. 15 , p. $249,2000$.

[12] B. Crone, A. Dodabalapur, A. Gelperin, L. Torsi, H. E. Katz, A. J. Lovinger, and Z. Bao, "Electronic sensing of vapors with organic transistors," Appl. Phys. Lett., vol. 78, p. 2229, 2001.

[13] J. T. Mabeck and G. G. Malliaras, "Chemical and biological sensors based on organic thin-film transistors," Anal. Bioanal. Chem., vol. 384, pp. 343-353, 2006.

[14] T. Someya, A. Dodabalapur, H. E. Gelperin, and Z. B. Katz, "Integration and response of organic electronics with aqueous microfluidics," Langmuir, vol. 18, pp. 5299-5302, 2002.

[15] D. H. Dunlap, P. E. Parris, and V. M. Kenkre, "Charge-dipole model for the universal field dependence of mobilities in molecularly doped polymers," Phys. Rev. Lett., vol. 77, p. 542, 1996.

[16] D. J. Gundlach, T. N. Jackson, D. G. Schlom, and S. F. Nelson, "Solvent-induced phase transition in thermally evaporated pentacene films," Appl. Phys. Lett., vol. 74, p. 3302, 1999.

[17] T. Someya, H. E. Katz, A. Gelperin, A. J. Lovinger, and A. Dodabalapur, "Vapor sensing with $\alpha, \omega$-dihexylquarterthiophene field-effect transistors: The role of grain boundaries," Appl. Phys. Lett., vol. 81, pp. 3079-3081, 2002b.

[18] L. Torsi, A. Dodabalapur, L. Sabbatini, and P. G. Zambonin, "Multi-parameter gas sensors based on organic thin-film-transistors," Sens. Actuatr B., vol. 67, pp. 312-316, 2000.

[19] J. Locklin and Z. Bao, "Effect of morphology on organic thin film transistor sensors," Anal. Bioanal. Chem., vol. 384, pp. 336-342, 2006.
[20] L. Wang, D. Fine, D. Sharma, L. Torsi, and A. Dodabalapur, "Nanoscale organic and polymeric field-effect transistors as chemical sensors," Anal. Bioanal. Chem., vol. 384, pp. 310-320, 2006.

[21] A. Assadi, G. Gustafsson, M. Willander, C. Svensson, and O. Inganas, "Determination of field-effect mobility of poly (3-hexylthiophene) upon exposure to NH3 gas," Synth. Met., vol. 37, pp. 123-130, 1990.

[22] R. M. Tiggelaar, T. T. Veenstra, R. G. P. Sanders, E. Berenschot, H. Gardeniers, M. Elwenspoek, A. Prak, R. Mateman, J. M. Wissink, and A. v. d. Berg, "Analysis system for the detection of ammonia based on micromachined components modular hybrid versus monolithicintegrated approach," Sens. Actuators B, vol. 92, pp. 25-36, 2003.

[23] A. Yimit, K. Itoh, and M. Murabayashi, "Detection of ammonia in the ppt range based on a composite optical waveguide $\mathrm{pH}$ sensor," Sens. Actuators B, vol. 88, pp. 239-245, 2003.

[24] T. Hutter and S. Ruschin, "Non-imaging optical method for multisensing of gases based on porous silicon," IEEE Sens. J., vol. 10, no. 1, pp. 97-103, Jan. 2010.

[25] F. Winquist, A. Spetz, and I. Lundström, "Determination of ammonia in air and aqeous samples with a gas-sensitive semiconductor capacitor," Anal. Chim. Acta, vol. 164, pp. 127-138, 1984.

[26] A. L. Kukla, Y. M. Shirshov, and S. A. Piletsky, "Ammonia sensors based on sensitive polyaniline films," Sens. Actuators B, vol. 37, pp. 135-140, 1996.

[27] V. V. Chabukswar, S. Pethkar, and A. A. Athawale, "Acrylic acid doped polyaniline as an ammonia sensor," Sens. Actuators B, vol. 77, pp. 657-663, 2001.

[28] J. W. Jeong, Y. D. Lee, Y. M. Kim, Y. W. Park, J. H. Choi, T. H. Park, C. D. Soo, S. M. Won, I. K. Han, and B. K. Ju, "The response characteristics of a gas sensor based on poly-3-hexylithiophene thin-film transistors," Sens. Actuators B, vol. 146, pp. 40-45, 2010.

[29] A. Torikai, M. Ohno, and K. Fueki, "Photodegradation of poly (methyl methacrylate) by monochromatic light: Quantum yield, effect of wavelengths, and light intensity," J. Appl. Polym. Sci., vol. 41, p. 1023, 1990.

[30] H.-W. Zan and K.-H. Yen, "High photoresponsivity of pentacene-based organic thin-film transistors with UV-treated PMMA dielectrics," Electrochem. Solid-State Letters, vol. 118, pp. H222-H225, 2008.

[31] C. Kim, A. Facchetti, and T. J. Marks, "Polymer gate dielectric surface viscoelasticity modulates pentacene transistor performance," Science, vol. 318, p. 76, 2007.

[32] H. Yan, Z. Chen, Y. Zheng, C. Newman, J. R. Quinn, F. Dötz, M. Kastler, and A. Facchetti, "A high-mobility electron-transporting polymer for printed transistors," Nature, vol. 457, p. 679, 2009.

[33] D. J. Gundlach, J. E. Royer, S. K. Park, S. Subramnian, O. D. Jurchescu, B. H. Hamadani, A. J. Moad, R. J. Kline, L. C. Teague, O. Kirillov, C. A. Richter, J. G. Kushmerick, L. J. Richter, S. R. Parkin, T. N. Jackson, and J. E. Anthony, "Contact-induced crystallinity for high-performance soluble acene-based transistors and circuits," Nature Mater., vol. 7, p. 216, 2008.

[34] A. Wang, I. Kymissis, V. Bulovic, and A. I. Akinwande, "Tunable threshold voltage and flatband voltage in pentacene field effect transistors," Appl. Phys. Lett., vol. 89, p. 112109, 2006.

[35] N. Benson, M. Schidleja, C. Melzer, R. Schmechel, and H. Von Seggern, "Complementary organic field effect transistors by ultraviolet dielectric interface modification," Appl. Phys. Lett., vol. 89, p. 182105, 2006.

[36] S. V. Novikov, D. H. Dunlap, V. M. Kenkre, P. E. Parris, and A. V. Vannikov, "Essential role of correlations in governing charge transport in disordered organic materials," Phys. Rev. Lett., vol. 81, p. 4472, 1998.

[37] G. Gu, M. G. Kane, J. E. Doty, and A. H. Firester, "Electron traps and hysteresis in pentacene-based organic thin-film transistors," Appl. Phys. Lett., vol. 87, p. 243512, 2005.

[38] A. R. Völkel, R. A. Street, and D. Knipp, "Carrier transport and density of state distributions in pentacene transistors," Phys. Rev. B, vol. 66, p. 195336, 2002.

[39] J.-B. Koo, C.-H. Ku, S.-C. Lim, S. H. Kim, and J. H. Lee, "Hysteresis and threshold voltage shift of pentacene thin-film transistors and inverters with AlO gate dielectric," Appl. Phys. Lett., vol. 90, p. 133503, 2007.

[40] H.-W. Zan and S.-C. Kao, "New organic phototransistor with biasmodulated photosensitivity and bias-enhanced memory effect," IEEE Trans. Electron Devices, vol. 30, no. 7, pp. 721-723, 2009.

[41] D. Li, E. J. Borkent, R. Nortrup, H. Moon, H. Katz, and Z. Bao, "Humidity effect on electrical performance of organic thin-film transistors," Appl. Phys. Lett., vol. 86, p. 042105, 2005. 


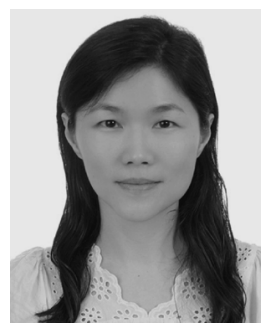

with nanostructures.
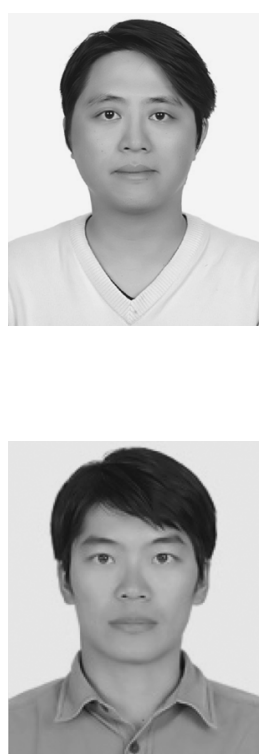

Yen-ren Lo received the B.S. degree in biology from Kaohsiung Medical University, Kaohsiung, the M.S. degree in biotechnology from National Dong Hwa University, Hualien. He is a Ph.D. student in National Chiao Tung University Institute of Bioinformatics and Systems Biology.

His research interests include bio-electronics interface and gas sensing.

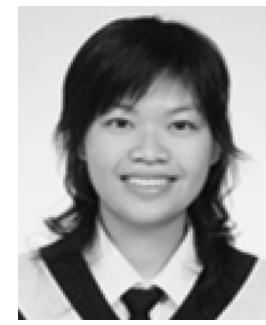

Yu-Mei Wu received both the B.S. and M.S. degrees in electro-optics from National Chiao Tung University, Hsinchu, Taiwan, and the M.S. degree in nanotechnology from Paris-Sud 11 University, Orsay, France, in 2010

Currently, she works in semiconductor industry about biosensor products in Hsinchu Science Park, Taiwan

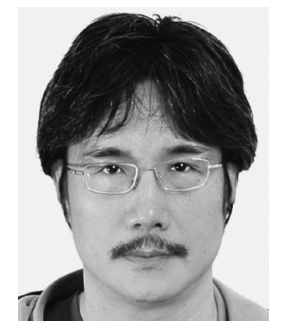

Yuh-Shyong Yang received the B.S. degree in forestry from National Taiwan University, the M.S degree in wood science and Technology from the University of California, Berkeley, and the Ph.D. degree in biochemistry from the University of Wisconsin, Madison, in 1979, 1983, and 1987, respectively.

He is currently a Professor of the Department of Biological Science and Technology, National Chiao Tung University. His research interests include enzymology, bioelectronics and protein engineering. 